\title{
Design of Model Predictive Controller for Pasteurization Process
}

\author{
Tesfaye Alamirew ${ }^{1}$, V. Balaji $i^{*}$, Nigus Gabbeye ${ }^{3}$ \\ ${ }^{1,3}$ Faculty of Chemical and Food Engineering, BIT, Bahir Dar University, Ethiopia \\ ${ }^{2}$ Faculty of Electrical and Computer Engineering, BIT, Bahir Dar University, Ethiopia \\ e-mail: balajieee79@gmail.com
}

\begin{abstract}
This research paper is about developing a better type of controller, known as MPC (Model Predictive Control) for pasteurization process plant. MPC is an advanced control strategy that uses the internal dynamic model of the process and a history of past control moves and a combination of many different technologies to predict the future plant output.. The dynamics of the pasteurization process was estimated by using system identification from the experimental data. The quality of model structures like $A R X, A R M A X, B J$ and $C T$ model structures was checked based on best fit with validation data, residual analysis and stability analysis. Auto-regressive with exogenous input (ARX322) model was chosen as a model structure of the pasteurization process dynamics and fits about $79.75 \%$ with validation data. Finally MPC control strategies were designed using ARX322 model structure.
\end{abstract}

Keywords: pasteurization process, MPC, ARX model, ARMAX model, BJ model, CT model

\section{Introduction}

In a modern world the economic and quality issues become more and more important, efficient control systems have become indispensable. Therefore the process industries require more reliable, accurate, robust, efficient and flexible control systems for the operation of process plant. In order to fulfill the above requirements there is a continuing need for research on improved forms of control. [1]

Control of temperature plays an important role in pasteurization plants. High temperature short time (HTST) is keeping milk or other food stuffs at $720 \mathrm{C}$ for 15 seconds in insulated holding tube. The pasteurization process consists of three stages like regeneration, heating and cooling sections. The crucial stage is heating process using heat exchanger to ensure unpasteurized product achieve desired pasteurization temperature before pass through holding tube and cooling sections. Prior to pasteurize milk sample, the equipment must have adequate controller to control the outlet temperature in order to maintain at standard value. [2]

The proportional integral (PI) and proportional integral derivative (PID) controllers are widely used in many industrial control systems because of its simple structure. These controllers are designed without process constraints only use mathematical expression based on error from a set point. In these circumstances, conventional controllers (PI and PID) are no longer to provide adequate and achievable control performance over the whole operating range. Thus designing a controller considering the process constraints and optimize the control performance is essential. [3]

Model Predictive Control also known as receding horizon control, is an advanced strategy for optimizing the performance of multivariable control systems. MPC generates control actions by optimizing an objective function repeatedly over a finite moving prediction horizon, within system constraints, and based on a model of the dynamic system to be controlled. [4]

\section{Process Description}

The plant PCT23, manufactured by Armfield (UK), is a laboratory version of a real industrial pasteurization process. It consists of a bench-mounted process unit to which is connected a dedicated control console. An interface card DT2811 is used for monitoring and controlling the process through a computer. [5] 


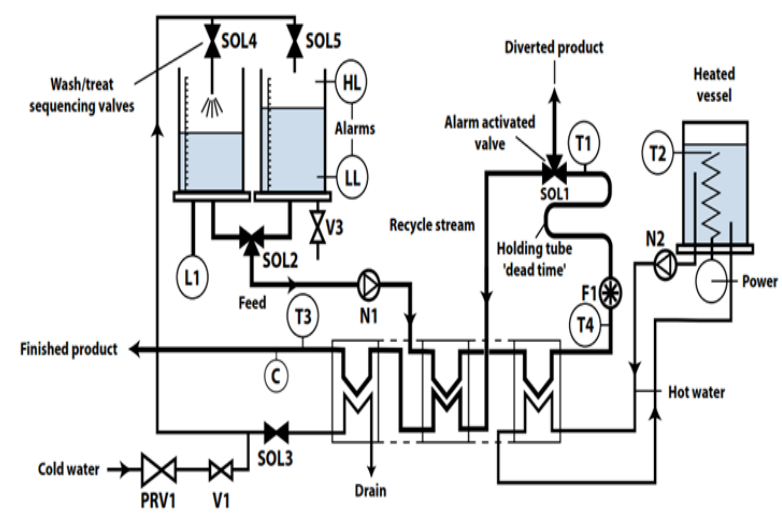

Figure 1. Process flow diagram of pasteurization plant

Here temperature $\mathrm{T} 1$ is the controlled variable and milk flow rate (N1), hot water flow rate (N2) and power are manipulated variables.

\section{Experimental Setup}

\subsection{Input-Output Data}

The input-output data was generated by introducing step input in milk flow rate, hot water flow rate and power input, then by recording pasteurization temperature response. The experiment was repeated two times for model estimation and validation purpose.

\subsection{System identification}

The input-output data was analyzed by the System Identification toolbox in MATLAB. The continuous and discrete model structures were tried to select the model structure that have best fit with validation. Then the selected model structure is tested for residual analysis and pole-zero analysis to check the model stability. The continuous time (CT) model, Autoregressive with exogenous input (ARX) model structures, ARMAX (auto regressive with moving average and exogenous (or extra) input model, and state space model structures were tried get best model structure in terms of best fit with validation data and model stability for further controller design. [6]

Best fit is calculated as:

$$
\text { Best fit }(\%)=\left(1-\frac{(y-\widehat{y})}{(y-\bar{y})}\right) * 100
$$

where: $\mathrm{y}$ is validation data, $\hat{\mathrm{y}}$ is estimated data and $\bar{y}$ is mean of validation data

After selection of best fit model structures model quality analysis like residual and pole zero location should be checked to select a nice and simple model for further controller design. The prediction error or residual is the key quantity.

It is defined as:

$$
\epsilon(\mathrm{k})=\mathrm{y}(\mathrm{k})-\hat{\mathrm{y}}(\mathrm{k})
$$

The stability of a system can be easily inferred by examining the pole locations of the transfer function. [7].

\subsection{Controller Design}

Controllers are basically employed in a closed loop control system. Closed loop control system is one that automatically changes the output based on the difference between the feedback signals to the input signal. Controller is an element used to produce manipulated variable from error variable, for Control action. [8][9]

IJEEI Vol. 5, No. 2, June 2017: $137-144$ 


\subsubsection{Model Predictive Control}

The model predictive uses quadratic minimization problem defined as:

$$
J=\left(y_{s p}-y\right)^{T}\left(y_{s p}-y\right) Q 1+\Delta u^{T} \Delta u Q 2
$$

Subject to: input and output constraints of the system. Where $y_{s p}$ is the set point, Q1 is output weight and Q2 is input weight. The size of this minimization function and weight matrixes are depend on prediction and control horizon. [10][11]

\section{Results and Discussions}

\subsection{Model Structure selection}

First step input was introduced at different time on milk flow rate, water flow rate and heater power to collect pasteurization temperature data with those three inputs. Two different experiments were done to collect the data for model estimation and validation purpose until it reaches to stability. The continuous time model fits $82.77 \%$ with the validation data better than the others. ARX422 (81.03\% fit), ARMAX3202 (80.9\% fit). The continuous time model doesn't mean a good model rather further analysis will be needed to select best model.

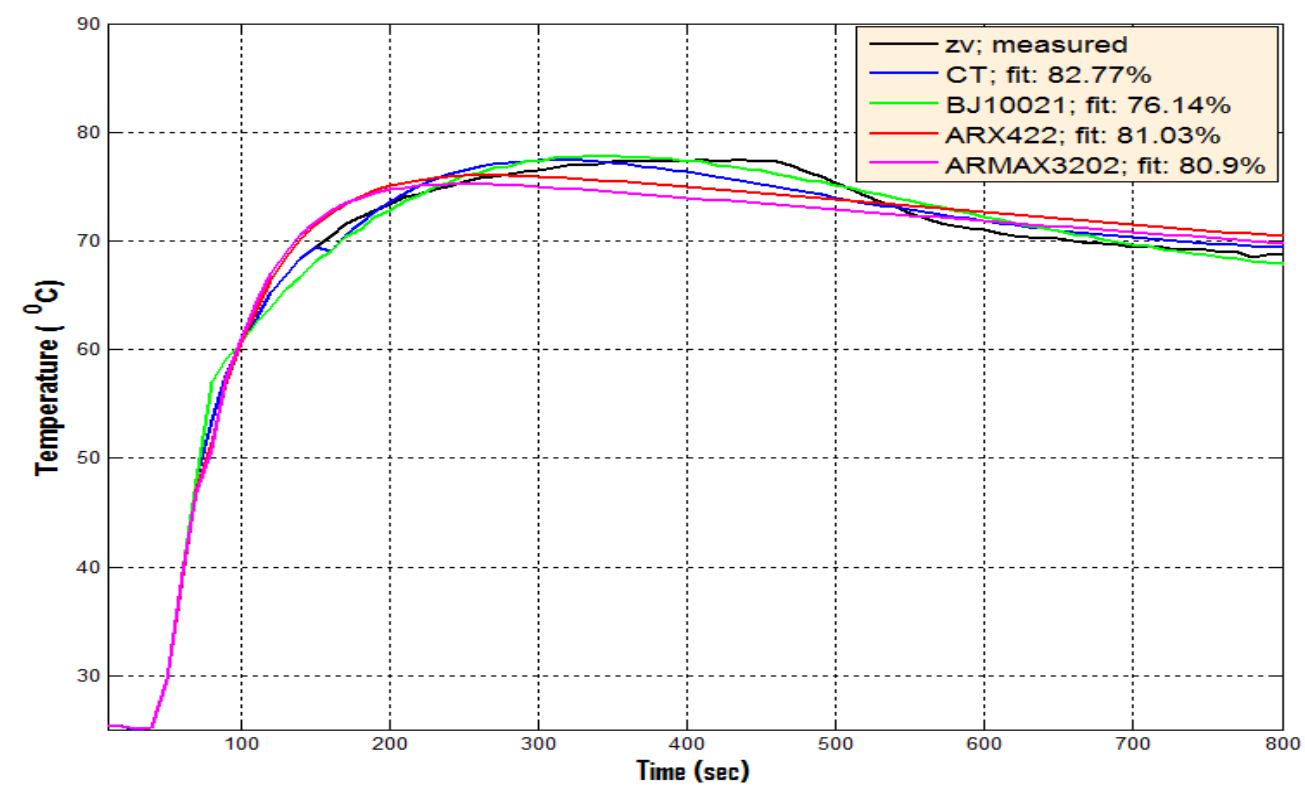

Figure 2. Percent fit of different model structures with validation data (zv).

\subsection{Model Quality Analysis}

\subsubsection{Residual Analysis}

For different model structures the auto corelation of residuals for the output (whitness test) and cross correlation of residuals with the input (independence test) were analyzed. From the graphs the horizontal scale is the number of lags, which is the time difference (in samples) between the signals at which the correlation is estimated. The upper and lower bounds on the plot represents the confidence interval of the corresponding estimates. Any fluctuations within the confidence interval are considered to be insignificant. A good model should have residuals uncorrelated with past inputs (independence test) and past outputs (whitness test). For poor models either auto and cross corelation residuals or two of residuals is out of the confidence region. In our case $99.9 \%$ confidence interval is taken. From Figure 3, the BJ10021 model is failed the analysis because both of auto and cross correlation residual analysis is out of the confidence region. The continuous time (CT) model also failed the analysis due to its auto correlation is out of the limit. ARMAX3202 and ARX422 models pass the residual analysis, but further analysis should be taken to select best model structure. 

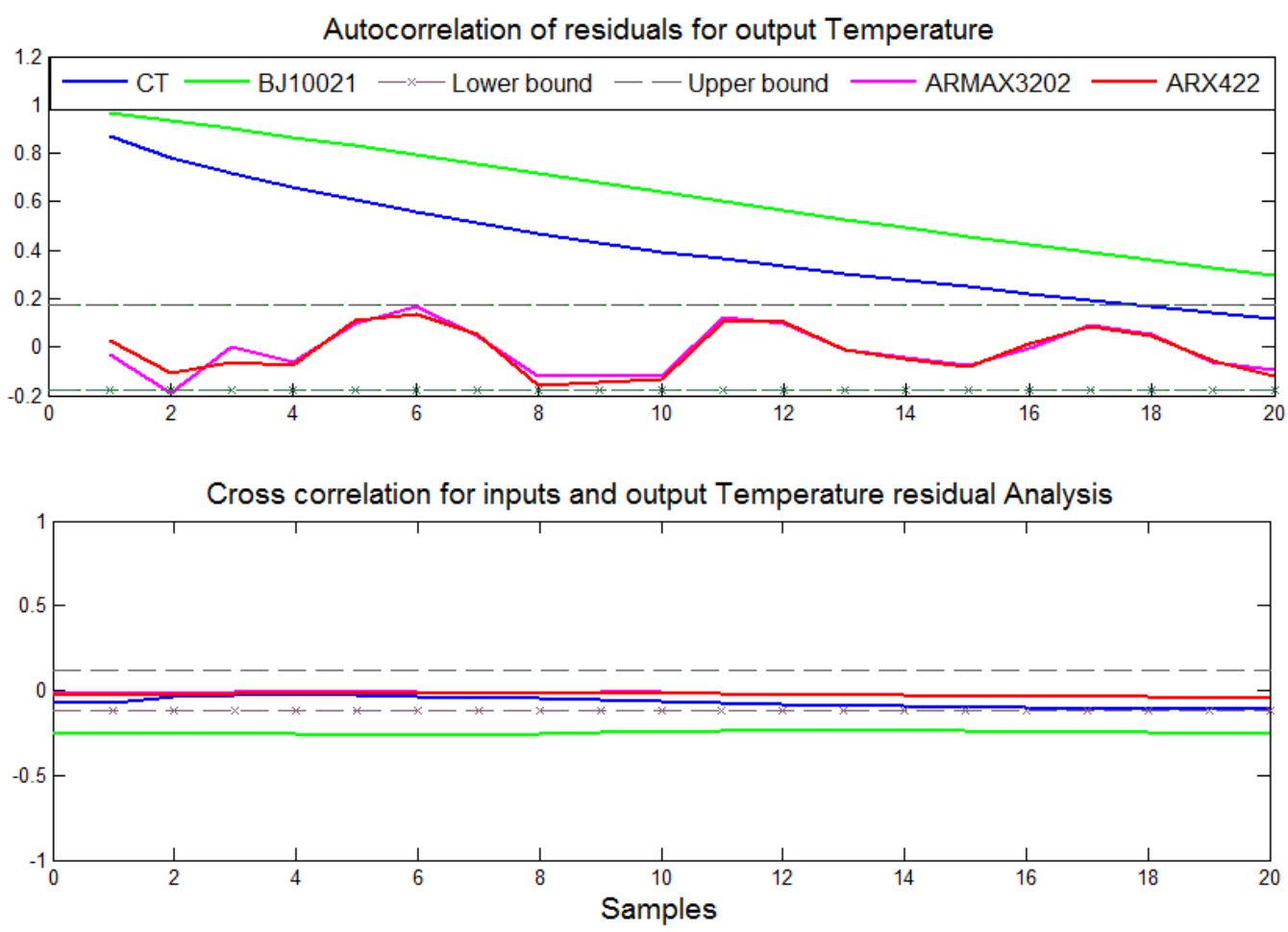

Figure 3. Residual analysis of model structures

\subsubsection{Stability Analysis}

The pole-zero location on the unit circle can tell as the stability of the process model. Poles are detrimental for the process stability. If all poles are inside a unit circle, the process model is stable. If one or more of its poles on unit circle, it is marginally stable. If one of its poles out of a unit circle, it is unstable.

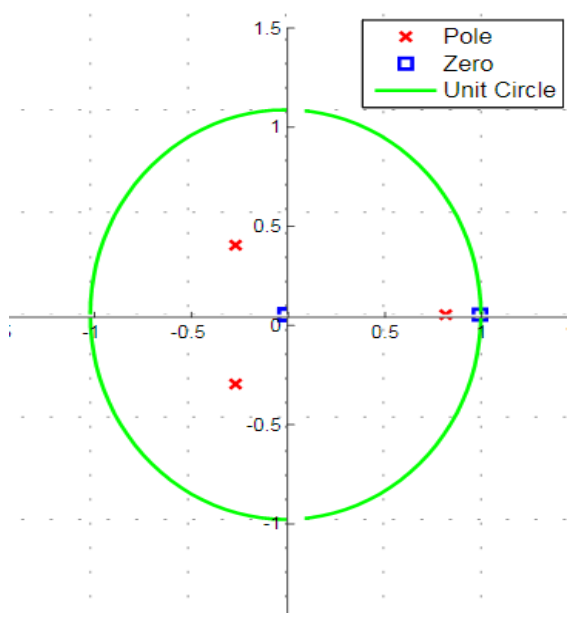

Figure 4. Pole-Zero location of ARX 422

When we see the pole-zero plot of the ARX422 model structure in Figure 4, it is stable because its entire pole is inside a unit circle in three of input - output relations. Some poles and zeros are lie on the same location. This means that we can cancel the numerator and 
denominator. Pole - zero cancelation may be an opportunity for model order reduction. Generally ARX422 model structure is stable and passes this analysis.

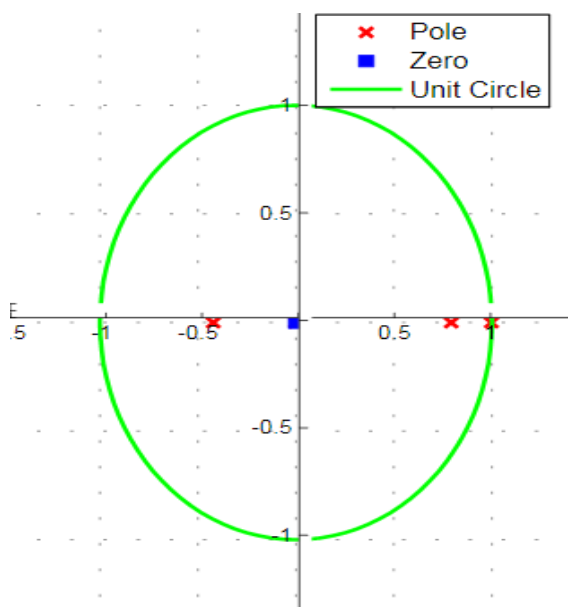

Figure 5. ARMAX3202 model structure pole-zero location

When we see Figure 5 one of its pole is on a unit circle, this means the process model is marginally stable. The process model is not selected because it has the chance to become unstable.

\subsubsection{Model Reduction}

When the model order reduced ARX gives slight decrease of fit.. When the ARX422 is reduced to ARX322 the final prediction error is 0.035. Therefore ARX322 can represent the model. Further reduction below this order deteriorates the fit percent with validation data. The reduced model also passes the model quality analysis. Therefore the ARX322 model represents the pasteurization process dynamics.

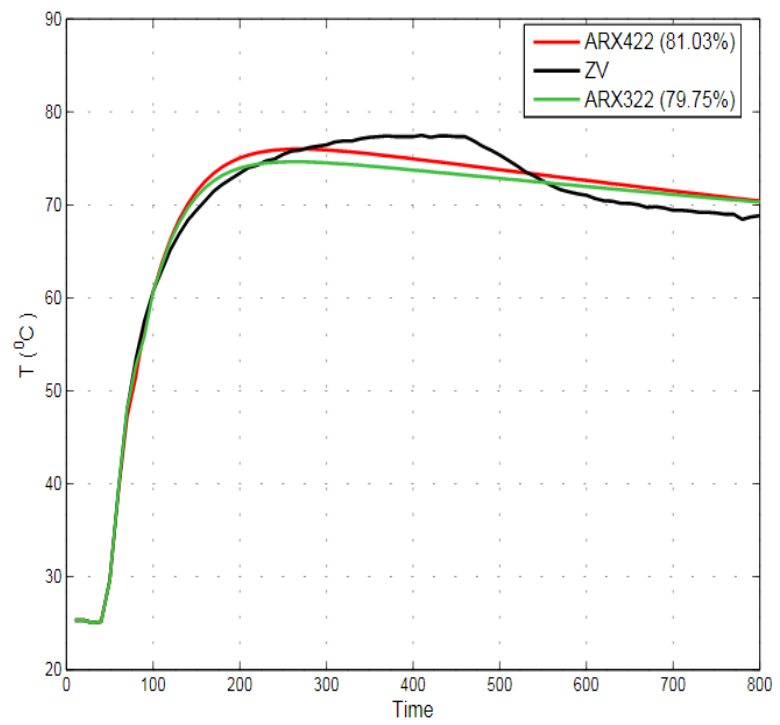

Figure 7. ARX422 and ARX322 model fit percent 
The following equation is the converted discrete ARX model to continuous dynamic model for PCT23 pasteurization plant dynamics. The process has three inputs that come parallel and one output. The process dynamics is the third order process and described as shown below.

Continuous-time ARX322 model is represented as follows.

$$
\mathrm{A}(\mathrm{s}) \mathrm{Y}(\mathrm{t})=\mathrm{B}(\mathrm{s}) \mathrm{u}(\mathrm{t})+\mathrm{C}(\mathrm{s}) \mathrm{e}(\mathrm{t})
$$
$5.55 * 10^{-7}$

Therefore the estimated parameters look like this $A(s)=s^{3}+0.105 s^{2}+0.0019 s+$

$$
\begin{aligned}
& \mathrm{B} 1(\mathrm{~s})=0.0007 \mathrm{~s}^{2}+5.22 * 10^{-5} \mathrm{~s}+5.98 * 10^{-8} \\
& \mathrm{~B} 2(\mathrm{~s})=0.0001 \mathrm{~s}^{2}+7.35 * 10^{-6} \mathrm{~s}-3.75 * 10^{-8} \\
& \mathrm{~B} 3(\mathrm{~s})=0.0072 \mathrm{~s}^{2}+0.0014 \mathrm{~s}+7.023 * 10^{-5} \\
& \mathrm{C}(\mathrm{s})=\mathrm{s}^{3}+0.22 \mathrm{~s}^{2}+0.019 \mathrm{~s}+0.00064
\end{aligned}
$$

Input delays are 10, 80 and 30 respectively for the three inputs (milk flow rate, hot water flow rate and power input). The ARX322 model has Loss function of 0.0334283 and final prediction error (FPE) of 0.034922 .

\subsection{MPC Controller Design}

Synthesis of MPC controller requires formation of an object's mathematical model, definition of cost function form and lengths of control and prediction horizons.

Lengthening of the prediction and control horizons elongates computation time, because it complicates optimization procedure. It also improves controller robustness. So there is a need to arbitrary and iteratively choose their lengths. The controller should be as robust to disturbances and as fast as possible.

The prediction horizon of the system should be large enough to cover the settling time.

The control horizon for different system should be different. It depends on the output signal of the system. In the most cases the control horizon should be large enough to get the reasonable stabilize output signal of the system.

\begin{tabular}{lcccc} 
Table1 : Model Predictive Controller parameter's values \\
\hline \multicolumn{1}{c}{ Parameter } & Value \\
\hline Prediction Horizon length & \multicolumn{2}{c}{200} & \\
Control Horizon length & 95 & \\
Matrix of Weights for the & & & & \\
Output Signal Q1 & 1 & 0 & $\ldots$ & 0 \\
& 0 & 1 & $\ldots$ & $\vdots$ \\
& $\vdots$ & $\vdots$ & $\ddots$ & 0 \\
& 0 & $\ldots$ & 0 & 1
\end{tabular}

Matrix of Weights for the Input Signal Q2

$$
\begin{array}{cccc}
0 & 0 & \ldots & 0 \\
0 & 0 & \ldots & \vdots \\
\vdots & \vdots & \ddots & 0 \\
0 & \ldots & 0 & 0
\end{array}
$$

\begin{tabular}{ll} 
Milk flow rate, mw [ml/min] & 326 \\
Hot water flow rate ,hw & $0<\mathrm{hw}<800$ \\
[ml/min) & $0<\mathrm{Pi}<1.7$ \\
\hline
\end{tabular}

Offline simulation for pasteurization temperature response is shown in Figure 8. The output response is reasonably tracts the set point without any overshoot, but it is slow (sluggish) because of it has too long control horizon. Working in optimal condition by considering process constraints makes MPC controller has best performance. 


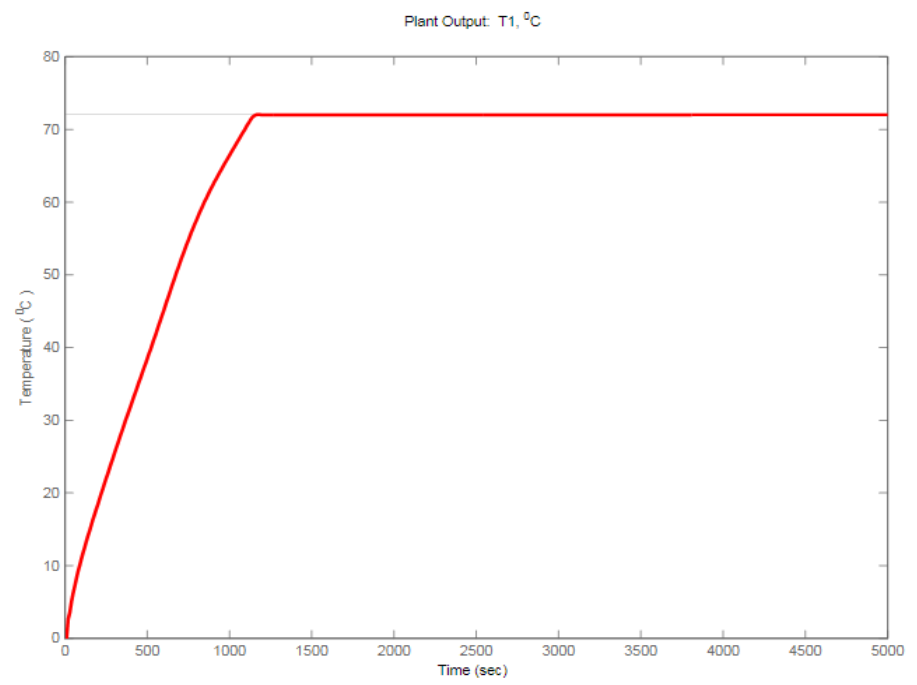

Figure 8. Pasteurization temperature response using MPC

\section{Conclusions}

Maintaining the temperature at a constant value is a critical issue in many of the Industries. MPC fulfills these types of difficulties by bringing the process variable to the desired set point as early as possible. MPC controller is more suitable for complex process control like milk pasteurization processes. From the simulation results, the MPC controller removes overshoot, but the control action is sluggish. to track set point immediately. This controller performance may be best, if it is used for real time pasteurization process environment.

\section{Acknowledgement}

The authors wish to thank Faculty of chemical and food engineering process control laboratory workers for their technical support and also, Mr. Zewdu Tsegaye and Mr. Zelfikir Jemal for their comments and suggestions.

\section{References}

[1] Diederich Hinrichsen, Anthony J Pritchard. Mathematical Systems Theory I. Modeling, State Space Analysis, Stability and Robustness. 2000; 1(6): 70.

[2] Negiz A, Cinar A, Schlesser JE, Ramanauskas P, Armstrong DJ, Stroup W. Automated control of high temperature short time pasteurization. Food Control 7. 1996: 309-315.

[3] Subhransu Padhee. Performance Evaluation of Different Conventional and intelligent. Sandviken, 80176 Gävle, Sweden.

[4] Orukpe PE. Basics of Model Predictive Control. Imperial College, London. April 14, 2005.

[5] ARMFIELD Process plant trainer (PCT23) Manual

[6] Lennart Ljung. System Identification: Theory for the user. Second Edition, Linköping University, Sweden. Prentice Hall PTR. 1999.

[7] Escobet, J Quevedo. Linear Model Identification Toolbox For Dynamic Systems. IEEE. 1998.

[8] Astrom, Karl J, Hagglund, Tore. Advanced PID Control, Department of Automatic Control, Lund Institute of Technology, Lund University.

[9] Yuvraj Bhusan Khare, Yaduvir Singh. PID control of Heat Exchanger System. Punjab, India, 2010.

[10] Sidharta Dash, Mihir Narayan Mohanty. Analysis of Outliers in System Identification using WMS algorithm. 2012.

[11] J Rossiter. Model-based predictive control: A Practical Approach. CRC Press LLC. 2003.

[12] MATLAB R2008b User Manual.

[13] F Yarman, BW Dickinson. Autoregredon Estimation Using Final Prediction Error. Proceedings of the IEEE. 1982; 70.

[14] Adriaan Van Den Bos. Parameter Estimation for Scientists and Engineers. Published by John Wiley \& Sons, Inc, Hoboken, New Jersey, 2007. 
[15] Garcia CE, Prett DM, Morari M. Model Predictive Control: Theory and Practice - a Survey. Automatica. 1989; 25(3): 335-348.

[16] Antonio Balsemin. Applications Oriented Input design for MPC: An analysis of a Quadruple water tank process. KTH Electrical Engineering degree project, Stockholm, Sweden. August 2012.

[17] Bequette, B Wayne. Process Control: Modeling, Design and Simulation. Prentice Hall PTR. 2002: 487-511.

[18] EC Kerrigan, JM Maciejowski. Soft constraints and exact penalty functions in Model predictive control. in Proc. UKACC International Conference. 2000. 